\title{
Analyses of alveolar epithelial injury via lipid-related stress in mammalian target of rapamycin inhibitor-induced lung disease
}

\author{
Nariaki Kokuho ${ }^{1,2} \cdot$ Yasuhiro Terasaki $^{1} \cdot$ Shinobu Kunugi ${ }^{1} \cdot$ Yoshinobu Saito $^{2} \cdot$ Hirokazu Urushiyama ${ }^{1}$. \\ Mika Terasaki ${ }^{1} \cdot$ Hiroki Hayashi $^{2} \cdot$ Akihiko Gemma $^{2} \cdot$ Akira Shimizu $^{1}$
}

Received: 17 February 2018 / Revised: 22 September 2018 / Accepted: 18 October 2018 / Published online: 6 February 2019

(c) United States \& Canadian Academy of Pathology 2019

\begin{abstract}
Although mammalian target of rapamycin inhibitors (mTORi) are used to treat various malignancies, they frequently induce active alveolitis and dyslipidemia. Abnormal lipid metabolism affects alveolar surfactant function and results in pulmonary disorders; however, the pathophysiology of lung injury and its relationship with lipid metabolism remain unknown. We investigated the relationship between lipid metabolism and alveolar epithelial injury, focusing on peroxisome proliferatoractivated receptor- $\gamma$ (PPAR- $\gamma$ ) as a lipid stress-related factor in mTORi-induced lung injury. We clinicopathologically examined three patients with mTORi-induced lung injury. We constructed an mTORi injury mouse model using temsirolimus in mice $(30 \mathrm{mg} / \mathrm{kg} / \mathrm{day})$, with the vehicle control and bleomycin injury groups. We also constructed a cultured alveolar epithelial cell injury model using temsirolimus $(0-40 \mu \mathrm{M})$ in the mouse lung epithelial cell line MLE-12 and performed analysis with or without pioglitazone (PPAR- $\gamma$ agonist) treatment. All three patients had dyslipidemia and lung lesions of hyperplastic pneumocytes with foamy and enlarged changes. In the mouse model, temsirolimus induced significantly higher levels of total cholesterol and free fatty acids in serum and higher levels of surfactant protein D in serum and BAL fluid with an increase in inflammatory cytokines in the lung compared to control. Temsirolimus also induced hyperplastic foamy pneumocytes with increased lipid-associated spots and larger round electron-lucent bodies compared to the control or bleomycin groups in microscopic analyses. Multiple lipid-associated spots within the cytoplasm were also induced by temsirolimus administration in MLE-12 cells. Temsirolimus downregulated PPAR- $\gamma$ expression in mouse lung and MLE-12 cells but upregulated cleaved caspase-3 in MLE-12 cells. Pioglitazone blocked the upregulated cleaved caspase-3 expression in MLE-12 cells. The pathogenesis of mTORi-induced lung disease may be involved in alveolar epithelial injury, via lipid metabolic stress associated with downregulated PPAR- $\gamma$ expression. Focusing on the relationship between lipid metabolic stress and alveolar epithelial injury represents a potentially novel approach to the study of pulmonary damage.
\end{abstract}

\section{Introduction}

The mammalian target of rapamycin (mTOR) is a serine/ threonine kinase located downstream of the phosphoinositide3 kinase-Akt signaling system and has a number of roles as a regulatory factor in the cell cycle, apoptosis, angiogenesis, and metabolism. Inhibitors of mTOR (mTORi) have drawn

Yasuhiro Terasaki

terasaki@nms.ac.jp

1 Department of Analytic Human Pathology, Graduate School of Medicine, Nippon Medical School, Tokyo, Japan

2 Department of Pulmonary Medicine and Oncology, Graduate School of Medicine, Nippon Medical School, Tokyo, Japan attention as antitumor agents, and their application in disease treatment has expanded not only to renal cell carcinoma and pancreatic neuroendocrine tumors but also to breast cancer and tumor lesions associated with tuberous sclerosis. Therefore, their frequency of use is expected to continue to increase. However, mTORi have serious side effects. In a retrospective chest computed tomography (CT) image evaluation after phase III trials in renal cell cancer patients administered temsirolimus (an mTORi), 29.2\% (52/178) of patients developed radiographically identified drug-related pneumonitis [1]. Moreover, lymphocytic alveolitis [2, 3], organizing pneumonia [4], and granulomatous formation [5] have been reported as phenotypes of mTORi-induced lung injury.

mTORi are also known to produce side effects, such as hypercholesterolemia and hypertriglyceridemia, through the 
suppression of adipogenesis in adipose tissue [6], and $>20 \%$ of patients in clinical trials exhibited dyslipidemia [7, 8].

Homeostasis of the intrapulmonary lipid environment is important for lung maintenance. The alveolar surfactant, which is the main component of the intrapulmonary lipid environment, is regulated by lipid absorption from the systemic circulation, in addition to the secretion, reuptake, reutilization, and catabolism of lipids in type II alveolar epithelium (ATII) cells [9]. In a study of 43 patients with dyslipidemia, several pulmonary gaseous exchange abnormalities, such as decreased arterial oxygen tension and carbon monoxide diffusion capacity, and the loss of ventilation/perfusion ratio distribution gradient have been reported [10]. It is also known that lipid droplets may appear in the cytoplasm as a result of cellular stress such as inflammation and oxidative stress in ATII cells [11]. Regarding the relationship between lipid metabolism abnormality and pulmonary fibrosis, some reports have indicated that fatty acid intake [12] and high free fatty acid levels are risk factors in the development of chronic pulmonary fibrosis [13]. Hence, it is suggested that abnormal lipid metabolism may affect the composition and function of alveolar surfactant and thus may be involved in the development of pulmonary fibrosis [14].

Lipid metabolic stress, such as alteration in the intrapulmonary lipid environment, is thought to be an important pathological condition in pulmonary disorders. Herein, we investigated the pathophysiology of alveolar epithelial injury induced by mTORi administration, focusing on the relationship to lipid metabolism abnormalities, through analysis of human cases, animal models, and cultured cells. Particular attention was paid to the foamy alveolar epithelium and lipid stress-related factors such as peroxisome proliferator-activated receptor- $\gamma$ (PPAR- $\gamma$ ), a lipid-related transcription factor located downstream of the mTOR pathway.

\section{Materials and methods}

\section{Patients}

All patients were diagnosed as having mTORi-induced lung injury by integrating clinical information, radiology images, and pathological findings in accordance with the diagnostic algorithms of interstitial lung disease [15]. Lung specimens were obtained by performing transbronchial lung biopsies at the Nippon Medical School Hospital (Tokyo, Japan) during 2011-2017. The patient characteristics can be found in Table 1. The study was approved by the Human Ethics Review Committee of Nippon Medical School and written informed consent was obtained from all patients prior to enrollment in the study (H26-11).
Table 1 Basic characteristics of three patients with mTORi-induced lung disease

\begin{tabular}{|c|c|c|c|}
\hline $\begin{array}{l}\text { Patients [normal } \\
\text { range] }\end{array}$ & Case 1 & Case 2 & Case 3 \\
\hline Age (years) & 58 & 71 & 57 \\
\hline Gender (M/F) & M & $\mathrm{F}$ & $\mathrm{F}$ \\
\hline Underlying disease & Renal cancer & Breast cancer & Breast cancer \\
\hline Drug & Everolimus & Everolimus & Everolimus \\
\hline $\begin{array}{l}\text { SP-D (ng/mL) } \\
{[0-109.9]}\end{array}$ & 157 & 119 & 203 \\
\hline TG (mg/dL) [30-149] & 261 & 93 & 246 \\
\hline $\begin{array}{l}\text { T-Cho }(\mathrm{mg} / \mathrm{dL}) \\
{[150-199]}\end{array}$ & 304 & 242 & 263 \\
\hline \multicolumn{4}{|l|}{ Bronchoalveolar lavage } \\
\hline $\begin{array}{l}\text { Total cell count } \\
\left(\times 10^{5}\right)\end{array}$ & 7.9 & 17.5 & N.E. \\
\hline $\begin{array}{l}\text { Alveolar } \\
\text { macrophages (\%) }\end{array}$ & 8 & 6 & 2 \\
\hline Lymphocytes (\%) & 80 & 94 & 96 \\
\hline Neutrophils (\%) & 0 & 0 & 0 \\
\hline Eosinophils (\%) & 12 & 0 & 2 \\
\hline
\end{tabular}

N.E. no evaluation

\section{Animal models}

Seven-week-old female C57BL/6 mice (Sankyo Labo Service Corporation Inc., Tokyo, Japan) were used in all experiments. Animals were randomized into groups before initiation of the experimental protocols, which were approved by the Animal Care and Use Committee of Nippon Medical School (27-013). Temsirolimus was purchased from Abcam (Cambridge, UK) and was suspended in $100 \%$ dimethyl sulfoxide (DMSO; Sigma, St. Louis, MO, USA) to a concentration of $30 \mathrm{mg} / \mathrm{mL}$ and stored at $-20{ }^{\circ} \mathrm{C}$ until use. Temsirolimus (10 mg kg//day) or vehicle was intraperitoneally administered 3 days/week for 4 weeks. The dose and dosing interval of temsirolimus were determined according to reports published elsewhere [16-18]. Bleomycin hydrochloride (BLM) was obtained from Nippon Kayaku (Tokyo, Japan). An osmotic pump (ALZET model 2001; DURECT Corporation, Cupertino, CA, USA) containing $200 \mu \mathrm{L}$ of saline with BLM $(100 \mathrm{mg} / \mathrm{kg})$ was implanted subcutaneously [19]. BLM was infused continuously via the pump for 14 days according to the manufacturer's instructions.

\section{Analysis of bronchoalveolar lavage (BAL) fluid (BALF) in mice}

Animals were anesthetized with a mixture of $2.0 \%$ isoflurane and $98 \%$ oxygen. Tracheas were cannulated and BAL was performed with $1.5 \mathrm{~mL}$ of normal saline solution. 
After counting the cell number in the BALF, the cells were cytospun onto glass slides and stained with Diff-Quick (Kokusai Shiyaku, Kobe, Japan) for differential cell counting.

\section{Biochemical analysis of serum and BALF in mice}

Enzymatic assays for total protein (TP), triglyceride (TG), free fatty acids (FFAs), and total cholesterol (T-Cho) were performed using LabAssay ${ }^{\mathrm{TM}}$ kits from Wako (Osaka, Japan). Surfactant protein (SP)-D levels were measured in serum and BALF according to the manufacturer's protocol using a specific Enzyme Immunoassay Kit from YAMASA (Tokyo, Japan), as described previously [20]. In brief, the kit contained a 96-well microtiter plate coated with monoclonal SP-D antibodies. The color change was measured spectrophotometrically at a wavelength of $450 \mathrm{~nm}$. The concentration of SP-D was determined by comparing the absorption of the samples to the standard curve.

\section{Micro-CT scan analysis}

To evaluate lung lesion injury, mice were anesthetized by inhalation of 1.0-2.0\% isoflurane (Pfizer Co., Tokyo, Japan) and subjected to CT imaging using a LaTheta LCT200 CT scanner (Hitachi Aloka Medical Ltd., Tokyo, Japan). Contiguous 1-mm slice images were assessed.

\section{Cell culture}

The lung epithelial cell line (MLE-12 cells) was purchased from the American Type Culture Collection (Manassas, VA, USA). MLE-12 cells were cultured in HITES medium, which contained Dulbecco's Modified Eagle's Medium Nutrient Mixture F-12 HAM (DME F12) supplemented with $2 \%$ fetal bovine serum, antibiotic $(100 \mathrm{U} / \mathrm{mL}$ penicillin), $1 \%$ ITS Liquid Media Supplement (insulin, transferrin, and sodium selenite), hydrocortisone $(10 \mathrm{nM})$, and $\beta$ estradiol $\left(10 \mathrm{nM}\right.$ ), at $37{ }^{\circ} \mathrm{C}$ and with $5 \% \mathrm{CO}_{2}$. DME F12 (lot RNBD6485), hydrocortisone (lot H6909), ITS (lot 13146), and estradiol (lot E2257) were obtained from Sigma. For western blotting, MLE-12 cells were seeded onto 6-well plates and treated with temsirolimus $(0-40 \mu \mathrm{M})$ for up to $24 \mathrm{~h}$. Pioglitazone (Wako) was dissolved in DMSO to prepare a primary stock solution of $25 \mathrm{mM}$ and stored at $-20{ }^{\circ} \mathrm{C}$. Temsirolimus $(20 \mu \mathrm{M})$ was administered with or without pioglitazone treatment $(10 \mu \mathrm{M})$ for $24 \mathrm{~h}$.

\section{Histologic examination of lung tissues}

Animals were anesthetized with a mixture of $2.0 \%$ isoflurane and $98 \%$ oxygen and euthanized by exsanguination, and samples were obtained. The lungs were fixed in $4 \%$ paraformaldehyde and embedded in paraffin. Lung sections $(3 \mu \mathrm{m})$ were stained with hematoxylin and eosin (HE) and examined under a light microscope.

\section{Immunohistochemistry}

Serial sections of deparaffinized, rehydrated lung tissues were washed with phosphate-buffered saline (PBS) and treated with $0.3 \% \mathrm{H}_{2} \mathrm{O}_{2}$ in methanol for 30 min to eliminate endogenous peroxidase activity. To unmask antigenic epitopes, sections were heated at $120^{\circ} \mathrm{C}$ for $20 \mathrm{~min}$ with $0.01 \mathrm{M}$ citrate buffer ( $\mathrm{pH}$ 6.0). Sections were then incubated with the appropriate dilution of primary antibody, polyclonal rabbit anti-cytokeratin, wide spectrum screening (DAKO, Glostrup, Denmark). Sections were then incubated with the Histofine Simple Stain Kit (Nichirei Biosciences, Tokyo, Japan) as the secondary antibody with peroxidase for $30 \mathrm{~min}$. Peroxidase activity was detected with a solution of 3,3'-diaminobenzidine and $\mathrm{H}_{2} \mathrm{O}_{2}$, with Mayer's hematoxylin as the counterstain.

\section{Sudan III staining}

Frozen lung sections were rinsed three times in distilled water. Slides were immersed for $2 \mathrm{~min}$ in $50 \%$ ethanol and incubated for $30 \mathrm{~min}$ in Sudan III solution (Wako) at $37^{\circ} \mathrm{C}$. Slides were then immersed in 50\% ethanol, rinsed twice in distilled water, and counterstained with Mayer's hematoxylin.

\section{Immunofluorescence staining of lung and cells}

\section{Pro-SP-C immunofluorescence and Nile Red staining in the lung}

Frozen lung sections were rehydrated in PBS and stained with an anti-pro-SP-C antibody (Hycult Biotech, Plymouth Meeting, PA, USA). Sections were rinsed three times with PBS and incubated with Donkey anti-Rabbit IgG, Alexa Fluor 488 (Invitrogen, Carlsbad, CA, USA) as the secondary antibody for $45 \mathrm{~min}$. The sections were then rinsed three times with PBS and counterstained for $10 \mathrm{~min}$ with $5 \mathrm{mg} /$ $\mathrm{mL}$ Nile Red (Wako) in 75\% aqueous glycerol and $15 \mathrm{~min}$ with Vectashield DAPI (4', 6'-diamidino-2-phenylindole; Vector Laboratories, Burlingame, CA, USA). Samples were analyzed by confocal fluorescence imaging microscopy using a DM6000-CFS TCS-SP5 (Leica, Tokyo, Japan). Cytoplasmic lipid-associated findings in MLE-12 cells were identified by Nile Red staining. After $10 \mu \mathrm{M}$ temsirolimus administration for $24 \mathrm{~h}$, MLE-12 cells were fixed in $4 \%$ paraformaldehyde for $20 \mathrm{~min}$ at room temperature. The cells were stained with $5 \mu \mathrm{g} / \mathrm{mL}$ Nile Red solution for $10 \mathrm{~min}$. The cells were rinsed three times with PBS after each 
manipulation, and the cells were stained and mounted with Vectashield DAPI for $15 \mathrm{~min}$. Samples were analyzed by confocal fluorescence imaging microscopy using a DM6000-CFS TCS-SP5 (Leica).

\section{Real-time quantitative reverse transcription-PCR amplification}

We analyzed PPAR- $\gamma$ mRNA expression by performing real-time RT-qPCR. Total RNA was purified from cells using an RNeasy Mini Kit (Qiagen Sciences, Germantown, MD, USA) according to the manufacturer's instructions. Reverse transcription was performed at $25^{\circ} \mathrm{C}$ for $10 \mathrm{~min}$, $37^{\circ} \mathrm{C}$ for $120 \mathrm{~min}$, and $85^{\circ} \mathrm{C}$ for $4 \mathrm{~min}$ with the High Capacity cDNA Reverse Transcription Kit (Applied Biosystems, Foster City, CA, USA). To detect mRNA, we used ready-to-use primer and probe sets from Applied Biosystems (Assays-on-Demand Gene Expression Catalog nos) as follows: PPAR- $\gamma, \# 1603599$ and Mm00440940_m1; glyceraldehyde 3-phosphate dehydrogenase (GAPDH), \#1579974 and Mm99999915_g1; interleukin (IL)-6, \#4453320 and Mn00446190_m1; IL-1 $\beta$, \#4453320 and Mn00434228_m1; matrix metalloproteinase (MMP)-9, \#1042604 and Mn00442991_m1; chemokine (C-C motif) ligand 2 (CCL2), \#4453320 and Mn00441242_m1; and tumor necrosis factor (TNF)- $\alpha$, \#4453320 and Mn443258. We optimized the primer and probe concentrations for each target gene by using TaqMan Universal PCR Master Mix (Applied Biosystems) according to the manufacturer's protocol. PCR $\left(2 \mathrm{~min}\right.$ at $50^{\circ} \mathrm{C}, 10 \mathrm{~min}$ at $95^{\circ} \mathrm{C}$, and 45 cycles of $15 \mathrm{~s}$ of denaturation at $95^{\circ} \mathrm{C}$ and $60 \mathrm{~s}$ of annealing at $60^{\circ} \mathrm{C}$ ) was performed with the ABI PRISM 7000 Sequence Detection System (Applied Biosystems) and fluorescent TaqMan methodology. To control for the variation in all experiments, gene expression levels of target sequences were normalized to the expression of GAPDH as an endogenous control. Relative gene expression data were calculated using the $2^{-\Delta \Delta \mathrm{Ct}}$ method [21].

\section{Western blotting}

TP from lung tissues or cells was extracted using a commercial kit (M-PER: Mammalian Protein Extraction Reagent; Thermo Scientific, Rockford, IL, USA) containing a protein-stabilizing cocktail (Halt Protease Inhibitor Cocktail; Thermo Scientific), $150 \mathrm{mM} \mathrm{NaCl}$, and $1 \mathrm{mM}$ EDTA. Homogenized samples were centrifuged $(15,000 \mathrm{rpm}, 25 \mathrm{~min})$, and the individual supernatants were termed lung and cell lysates, respectively. Then $15 \mu \mathrm{L}$ of sodium dodecyl sulfate (SDS) sample buffer containing 2mercaptoethanol for reduction was added to an equal volume of culture medium or lysate sample. These samples were boiled at $95{ }^{\circ} \mathrm{C}$ for $5 \mathrm{~min}$, subjected to SDS- polyacrylamide gel electrophoresis on a $10 \%$ acrylamide separating gel (e-PAGEL; ATTO, Tokyo, Japan), and transferred to polyvinylidene difluoride membranes (Invitrogen) using an electroblot apparatus (Invitrogen). Membranes were incubated for $1 \mathrm{~h}$ at room temperature with protein-free T20 Tris-buffered saline blocking buffer (Thermo Scientific), and then incubated at $4{ }^{\circ} \mathrm{C}$ overnight with antibody against phosphorylated-mTOR (p-mTOR) (Abcam) at a dilution of 1:1000, PPAR- $\gamma$ (CST, Beverly, MA, USA) at a dilution of 1:1000, cleaved caspase-3 (CST) at a dilution of 1:1000, and GAPDH (Epitomics, Burlingame, CA, USA) at a dilution of 1:30,000. After the membranes were washed several times with Tris-buffered saline containing $0.1 \%$ Tween 20 , they were incubated with the appropriate horseradish peroxidase-conjugated secondary antibody (Promega, Madison, WI, USA) for $60 \mathrm{~min}$, washed with Tris-buffered saline containing $0.1 \%$ Tween 20, and developed with SuperSignal West Femto Luminol/ Enhancer solution (Thermo Scientific). Immunoreactivity of the blots was detected with an LAS-4000 Luminescent Image Analyzer with a CCD Camera (Fujifilm, Tokyo, Japan).

\section{Electron microscopy}

Small pieces of lung tissue from each sample were fixed in $2.5 \%$ glutaraldehyde, embedded using a few drops of epoxy resin (Epok 812; Oken C., Ltd., Tokyo, Japan) mixture, cut into $500 \mathrm{~nm}$ sections, and stained with uranyl acetate and lead citrate. The grids were examined with an H-7500 electron microscope (Hitachi, Tokyo, Japan) operating at $80 \mathrm{kV}$.

\section{Statistical analysis}

Two-group comparisons were analyzed using an unpaired Student's $t$ test, and multiple-group comparisons were performed with a one-way analysis of variance followed by Tukey's post hoc analysis, and values were reported as mean \pm standard error. $P$ values $<0.05$ were considered to be significant. All analyses were conducted using the JMP software, version 12 for Windows (SAS Institute Inc., Cary, NC, USA).

\section{Results}

\section{mTORi induced hyperlipidemia and foamy alveolitis in human subjects}

We performed clinicopathological evaluations of three cases with mTORi-induced lung injury admitted to our hospital. All patients had complications of dyslipidemia and 
Fig. 1 Histologic features of lung lesions obtained by transbronchial lung biopsy in three patients with mTORiinduced lung disease. Hyperplastic pneumocytes with foamy and enlarged changes were observed with hematoxylin-eosin staining (af) and cytokeratin staining (g-i). d-f High-magnification views of the rectangular areas in $\mathbf{a}-\mathbf{c}$, respectively. Arrowheads indicate foamy and enlarged pneumocytes. Scale bars are $200 \mu \mathrm{m}(\mathbf{a}-\mathbf{c})$ and $20 \mu \mathrm{m}(\mathbf{d}-\mathbf{i})$
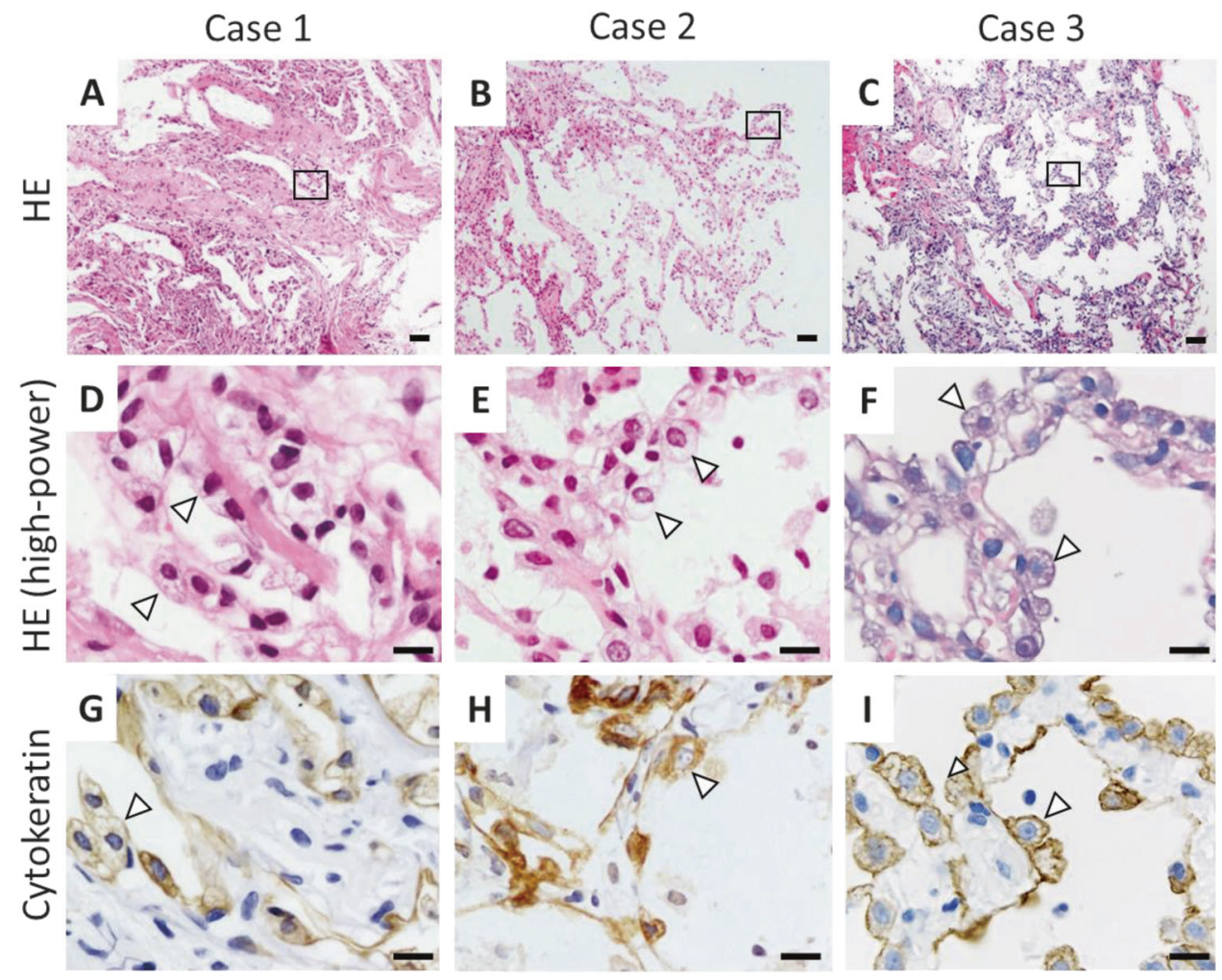

showed high values of serum SP-D, and lymphocyte subsets in the cellular fraction of BALF were above the normal range (Table 1). Pathological findings in all patients revealed lymphocytic alveolitis with hyperplasia of foamy and enlarged pneumocytes (Fig. 1, arrows).

\section{mTORi induced hyperlipidemia and foamy alveolitis in mice}

In the present study, we compared mTORi mice with a normal group (control mice) and an injury group treated with bleomycin hydrochloride (BLM mice), which causes marked inflammation and epithelial injury in the lung [22], as mTORi-induced lung injury was relatively mild in the human subjects (see Fig. 1).

There were no significant differences in serum TP levels (Fig. 2a), whereas serum T-Cho $(P<0.01$ and $P<0.01)$ and FFAs $(P<0.05$ and $P<0.01)$ showed significantly higher values in mTORi mice than in control and BLM mice (Fig. 2c, d), respectively; thus, as in the human subjects, abnormal lipid metabolism was observed in mTORi mice.

Micro-CT in mTORi mice showed a focal hyperintense lesion (Fig. 3b, circle), which is related to the pathological findings of a large number of foamy cells in the alveolar space and ATII cell hyperplasia showing SP-C- and Ki-67positive staining (Fig. 3e, h, k, n). In BLM mice, micro-CT showed a diffuse hyperintense lesion associated with pathological findings of alveolar wall thickening with
A
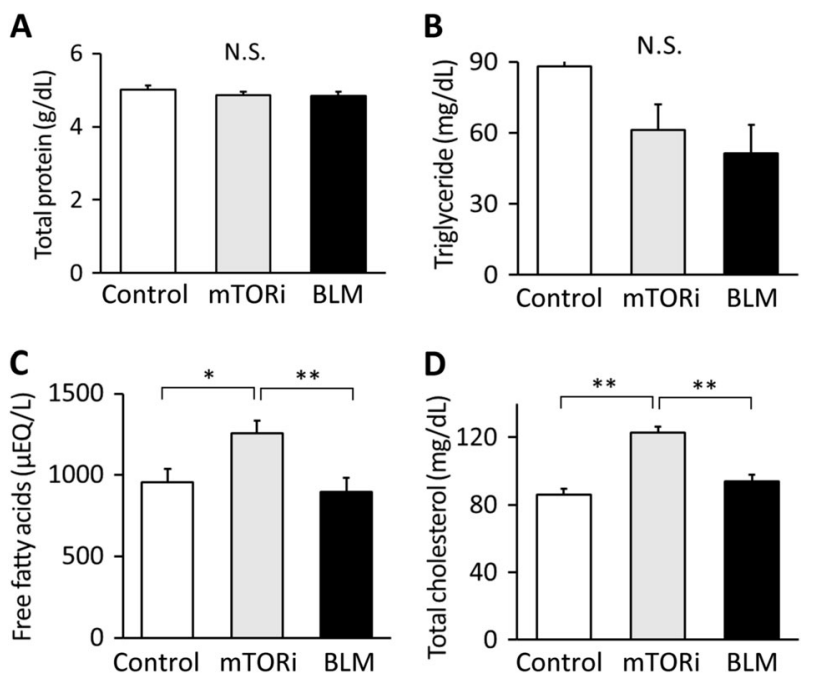

Fig. 2 mTORi induces systemic lipid metabolic disorder in mice. a-d The serum values of total protein, triglyceride, free fatty acids, and total cholesterol in control, mTORi, and BLM mice. $(n=6,6$, and 5, respectively). Data are shown as the mean \pm S.E. N.S. not significant. $* P<0.05$ and $* * P<0.01$ versus mTORi mice

marked inflammatory cell infiltration and hyperplasia of ATII cells (Fig. 3c, f, i, 1, o). The total cell count in BALF of mTORi mice was significantly higher than that of control mice (Fig. 3p), and the cell fraction analysis by Diff-Quick staining revealed that the percentage of lymphocytes was significantly higher than in control mice (Fig. 3q). The protein concentration in BALF of mTORi mice was 
Fig. 3 mTORi induces alveolar epithelial injury in mice. a-c Chest micro-CT axial sections of control, mTORi, and BLM mice, respectively. $\mathbf{d}-\mathbf{i}$ Lung sections for each group were stained with hematoxylin-eosin. g-i Highmagnification views of $\mathbf{d}-\mathbf{f}$, respectively. $\mathbf{j}-\mathbf{o}$ Lung sections for each group were stained for SP-C (j-l) or Ki-67 (m-o). Scale bars are $200 \mu \mathrm{m}(\mathbf{d}-\mathbf{f})$ and $20 \mu \mathrm{m}$ $(\mathbf{g}-\mathbf{o}) . \mathbf{p}, \mathbf{q}$ Total cell count and percentage of cell fraction in bronchoalveolar lavage fluid (BALF) for each group $(n=4$, 11 , and 10 , respectively). $\mathbf{r}$ Protein levels in BALF for each group $(n=7,15$, and 13 , respectively). $\mathbf{s}, \mathbf{t}$ SP-D levels in $\operatorname{BALF}(n=6,9$, and 9 , respectively) or serum $(n=10$, 23 , and 12 , respectively) for each group. Data are shown as the mean \pm S.E. N.S. not significant. $* P<0.05$ and $* * P<$ 0.01 versus mTORi mice
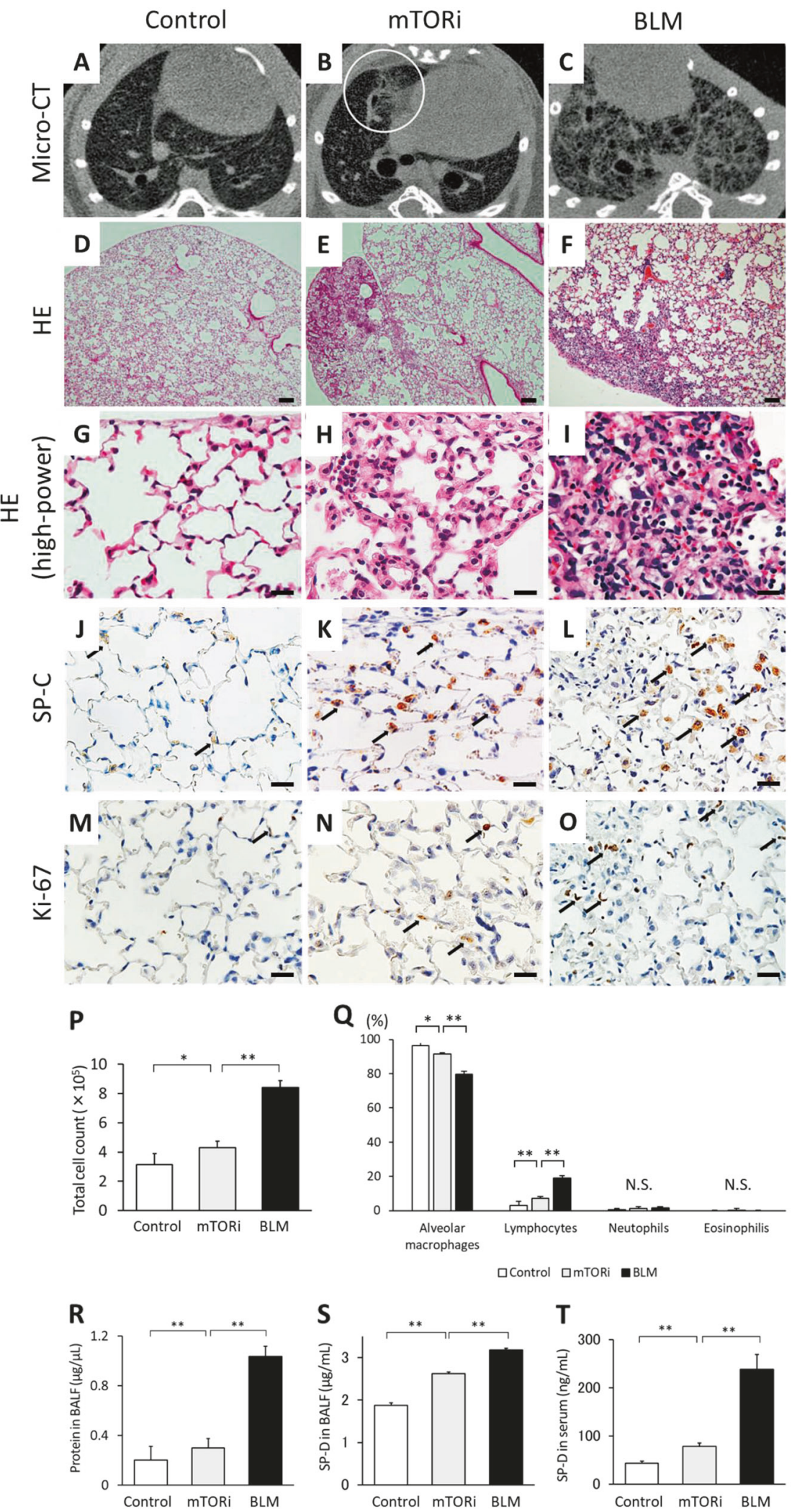

significantly higher than in control mice (Fig. 3r). SP-D levels in BALF and serum of mTORi mice were significantly higher than in control mice (Fig. 3s, t). These results suggest that mTORi promoted capillary-alveolar permeability and induced alveolar epithelial injury with lymphocytic infiltration in the mouse lung. BLM mice 
showed higher values in total cell count, ratio of lymphocytes, protein concentration, and SP-D level in BALF and serum compared to those of mTORi mice (Fig. 3p-t). Thus our mTORi mouse model appeared similar to the human cases of mTORi-induced injury, evidenced by our data showing that lung injuries in humans and mouse models administered mTORi were both related to serum lipid metabolic abnormalities, high serum SP-D values, and lymphocytic alveolitis with foamy pneumocyte hyperplasia and foamy macrophage accumulation in alveoli.

Although mTORi induced relatively mild alveolar epithelial injury compared to BLM with respect to the level of
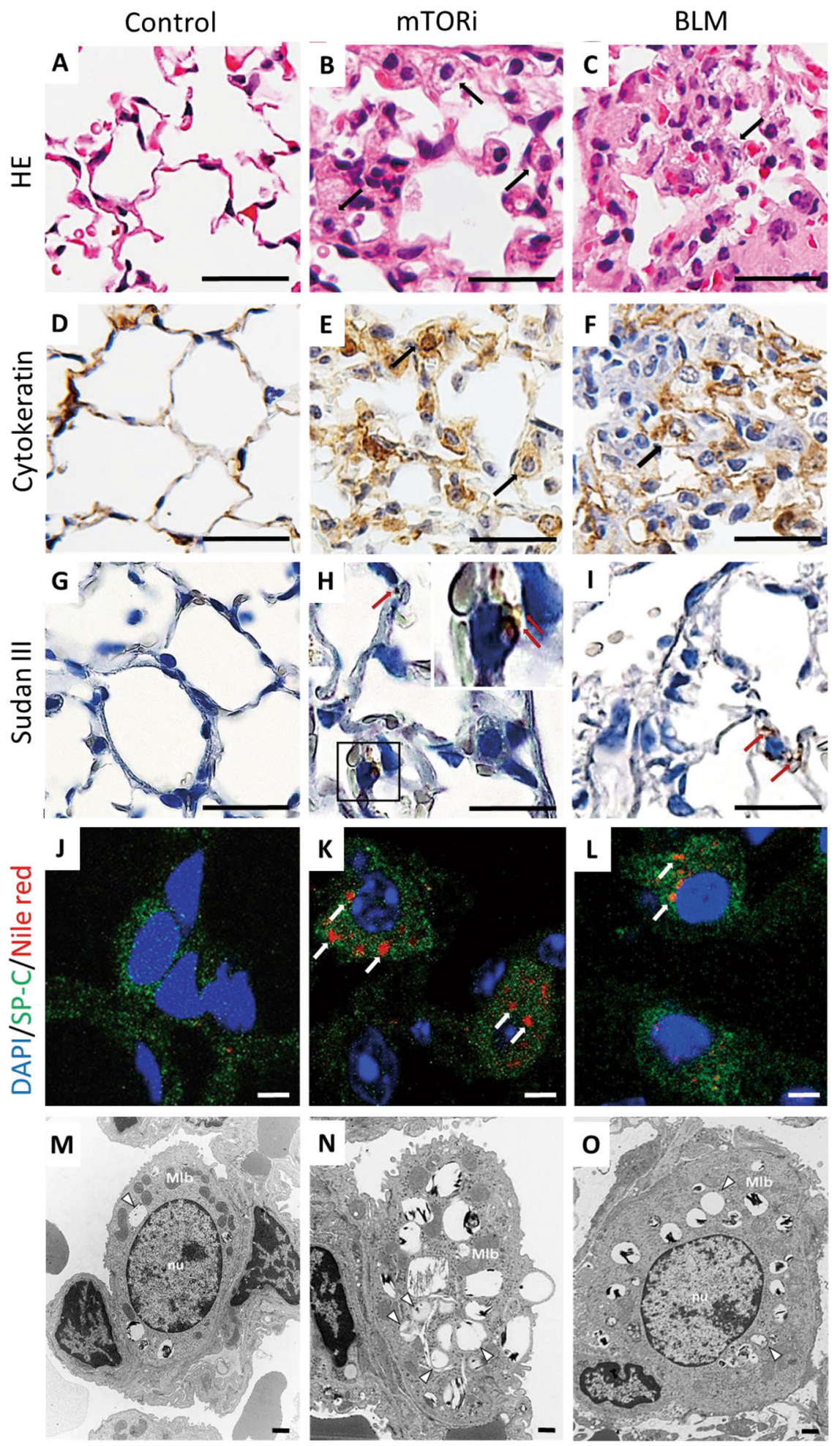

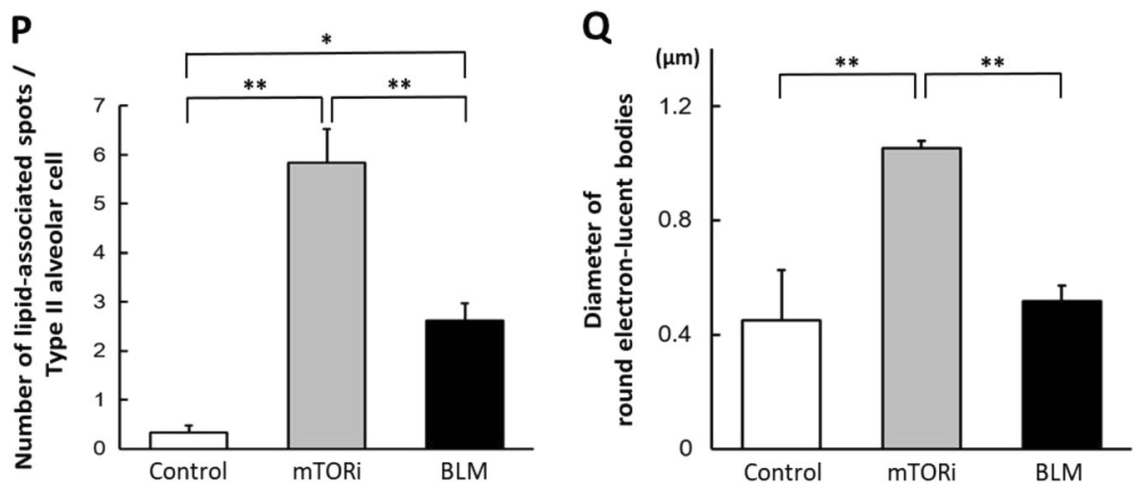

Fig. 4 mTORi induces emergence of lipid-associated vacuoles within the cytoplasm of pneumocytes in mice. a-f Lung sections for each group were stained with hematoxylin-eosin $(\mathbf{a}-\mathbf{c})$ and cytokeratin $(\mathbf{d}-\mathbf{f})$. Arrows indicate hyperplastic enlarged foamy pneumocytes. $\mathbf{g}-\mathbf{i}$ Lung sections for each group were stained with Sudan III. Inset: high-magnification view of area in the rectangle. Arrows indicate lipid-associated spots in pneumocytes. $\mathbf{j}-\mathbf{l}$ Lung sections for each group were subjected to fluorescent triple staining using SP-C (green), Nile Red (red), and DAPI (blue). Arrows indicate lipid-associated spots within ATII cells. m-o Representative electron microscopic images in each group showing the structure of ATII cells. nu nucleus, Mlb mitochondria-like body, round electron-lucent bodies (arrowheads). Scale bars are $20 \mu \mathrm{m}(\mathbf{a}-\mathbf{i}), 5 \mu \mathrm{m}(\mathbf{j}-\mathbf{l})$, and $0.5 \mu \mathrm{m}$ $(\mathbf{m}-\mathbf{0})$. $\mathbf{p}$ The number of lipid-associated spots within the cytoplasm of ATII cells in each group $(\mathbf{j}-\mathbf{l})$. The red spots $(1 \mu \mathrm{m}$ or more $)$ within the cytoplasm of ATII cells $(n=15)$ were counted in each group $(n=3)$. q The diameter of round electron-lucent bodies within the cytoplasm of ATII cells $(n=30)$ was measured in each group $(n=3)(\mathbf{m}-\mathbf{0})$. Data are shown as the mean \pm S.E. $* P<0.05$ and $* * P<0.01$

damage, the lung pathological findings in mTORi mice showed notable foamy changes of enlarged pneumocytes as well as macrophages, according to $\mathrm{HE}$ and cytokeratin staining (Fig. 4a-f). Thus we performed Sudan III staining and fluorescent staining using Nile Red and SP-C immunohistochemistry to analyze the components of foamy alveolar epithelial cells in the three mouse groups (Fig. 4g-1). The results showed that the number of lipidassociated spots within pneumocytes in mTORi mice was significantly higher than that of control or BLM mice (Fig. $4 \mathrm{j}-1, \mathrm{p}$ ). We also performed an electron microscopic study to analyze the size of round electron-lucent bodies within pneumocytes in mTORi mice. The size of electronlucent bodies within pneumocytes in mTORi mice was significantly larger than control or BLM mice (Fig. 4m-o, q). Thus, while the findings of pneumocyte damage in mTORi mice are relatively mild, the notable appearance of lipid-associated spots in pneumocytes seems to be characteristic.

\section{mTORi increased inflammatory cytokine expression and downregulated PPAR- $\gamma$ protein expression in the mouse lung}

We evaluated the mRNA expression of various inflammatory cytokines in the mouse lung and found that the mRNA expression of IL-1 $\beta$, IL-6, MMP-9, CCL2, and TNF- $\alpha$ was upregulated in the lung homogenates of mice treated with temsirolimus (Fig. 5a-e).

mTOR forms the protein complex termed mTOR complex 1 (mTORC1) in its signaling pathway, while PPAR- $\gamma$, which regulates the synthesis and absorption of fatty acids, exists downstream of mTORC1 and is thus regulated specifically by mTORC1 [23]. Consistent with this pathway, we found that PPAR- $\gamma$ protein expression in the whole lung of mTORi mice was downregulated compared to control mice, attributable to suppressed mTOR phosphorylation by mTORi and that confirmed the inhibitory effect of temsirolimus as an mTORi (Fig. 5f-h). Thus the downregulated PPAR- $\gamma$ expression may be involved in the pulmonary inflammation observed in mTORi mice.

\section{mTORi induced lipid-associated spots with altered PPAR- $\gamma$ and cleaved caspase-3 expression in MLE-12 cells}

In order to confirm the observed characteristics of pneumocytes in the lung tissue of mTORi mice, we performed analyses using lung epithelial model cells (MLE-12 cells) [24].

Similar to mTORi mice, analysis of fluorescence staining for lipids using Nile Red showed a large number of lipidassociated spots in the cytoplasm of MLE-12 cells following mTORi administration (Fig. 6a).

Furthermore, PPAR- $\gamma$ mRNA expression in MLE-12 cells following mTORi treatment was downregulated compared to control (without mTORi) (Fig. 6b). Moreover, PPAR- $\gamma$ protein expression in MLE-12 cells was downregulated in both a dose $(2-40 \mu \mathrm{M})$ and time $(0.5-24 \mathrm{~h})$ dependent manner. In contrast, the protein expression of cleaved caspase-3, which is activated during cell apoptosis, in MLE-12 cells was upregulated in a dose- and timedependent manner (Fig. 6c). Thus our data suggested that mTORi induced lung epithelial injury through the PPAR- $\gamma$ pathway in mice and MLE-12 cells, as evidenced by the 
Fig. 5 mTORi increases inflammatory cytokine expression and downregulates PPAR- $\gamma$ protein expression in mouse lung. a-e Quantitative mRNA expression of IL- $1 \beta$, IL6, MMP-9, CCL2, and TNF- $\alpha$ in the lung homogenate of control and temsirolimus-treated mice ( $n=5$ and 7 , respectively). $\mathbf{f}$ Western blot analysis of the expression of phosphorylatedmTOR (p-mTOR) and PPAR- $\gamma$ in the lung of mice at Day 28 after temsirolimus treatment. $\mathbf{g}$, h Densitometric analysis of western blot bands, normalized to GAPDH $(n=3)$. Data are shown as the mean \pm S.E. $* P<$ 0.05 and $* * P<0.01$
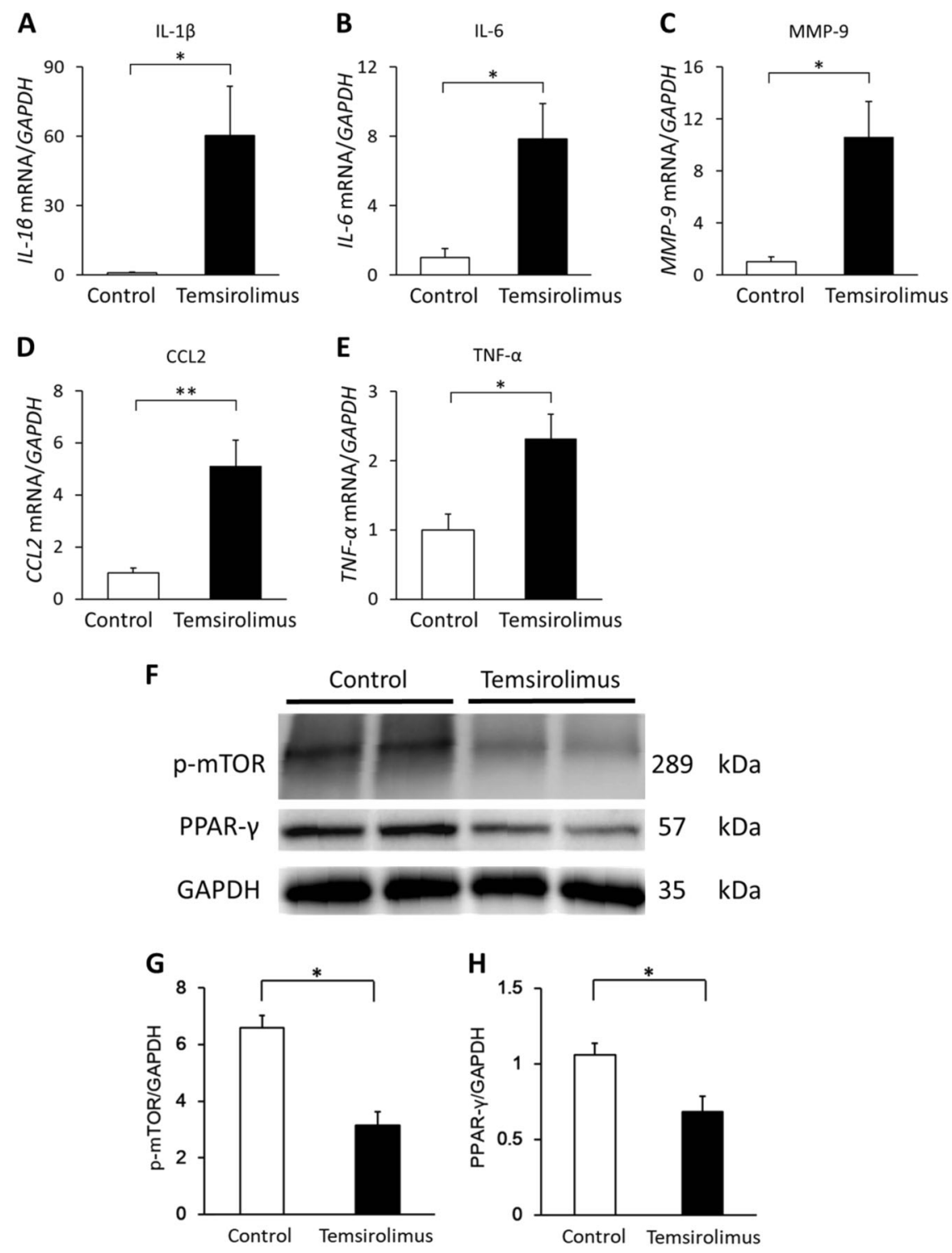

Temsirolimus

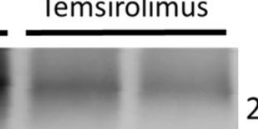

$289 \mathrm{kDa}$

$57 \mathrm{kDa}$

$35 \mathrm{kDa}$

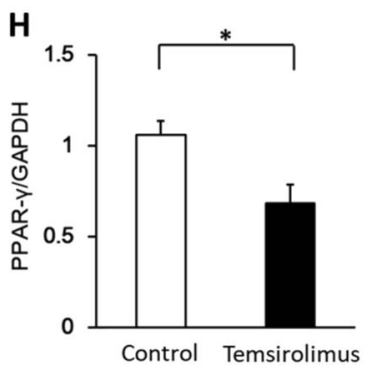

associated emergence of lipid-associated spots. We then evaluated the change in lipid-associated spot emergence and cleaved caspase- 3 expression by mTORi under treatment with the PPAR- $\gamma$ agonist pioglitazone in MLE-12 cells. We found that the upregulation of lipid-associated spot emergence and cleaved caspase- 3 expression following mTORi administration was inhibited by treatment with pioglitazone (Fig. 7a-c).

\section{Discussion}

Lipid metabolic stress is considered to be an important pathological condition in various pulmonary disorders. In the present study, we investigated the pathophysiology of lung injury caused by mTORi administration using human subjects, mouse models, and a lung epithelial model cell line, focusing on foamy changes of the alveolar epithelium and lipid stress-related factors.

Our results showed that mTORi induced dyslipidemia and alveolar epithelial injury, accompanied by the emergence of foamy changes and lipid-associated vacuoles within pneumocytes, in both human and mouse models, which was suggested to be associated with local (i.e., alveolar epithelium) lipid metabolic stress and systemic lipid metabolic stress caused by mTORi. We also found that alveolar epithelial injury could be induced via lipid metabolic stress due to reduced expression of PPAR- $\gamma$, which is a lipid-related transcription factor located downstream of the mTORC1 pathway. Thus we discuss the relationship between lipid metabolic stress and alveolar epithelial injury.

Our data showed that lung injury in both human and mouse models administered mTORi was related to serum lipid metabolic abnormalities, high serum SP-D values, and 
Fig. 6 Effects of mTORi administration on a lung epithelial cell line. a Fluorescence staining of MLE12 cells with Nile Red (red) and DAPI (blue) $24 \mathrm{~h}$ after temsirolimus treatment $(20 \mu \mathrm{M})$. Scale bars are $5 \mu \mathrm{m}$. b Quantitative PPAR- $\gamma$ mRNA expression in MLE-12 cells 30 min after temsirolimus treatment $(20 \mu \mathrm{M})(n=3)$. Data are shown as the mean \pm S.E. $* * P<0.01$. c Western blot analysis of the expression of phosphorylated-mTOR (pmTOR), PPAR- $\gamma$, and cleaved caspase-3 in MLE-12 cells cultured for up to $24 \mathrm{~h}$ after temsirolimus treatment $(0-20 \mu \mathrm{M})$
A

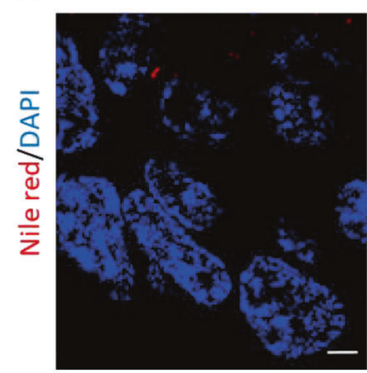

Control

C

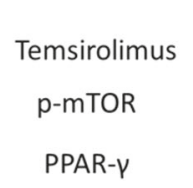

Cleaved caspase-3

GAPDH
B

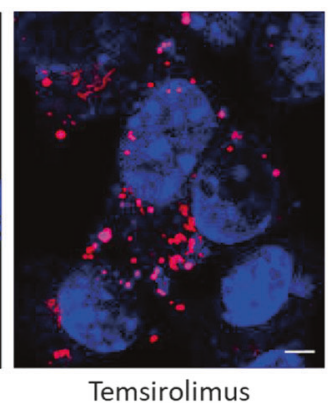

Temsirolimus

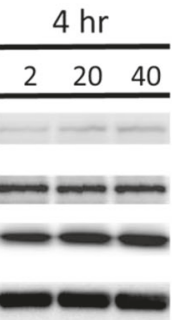

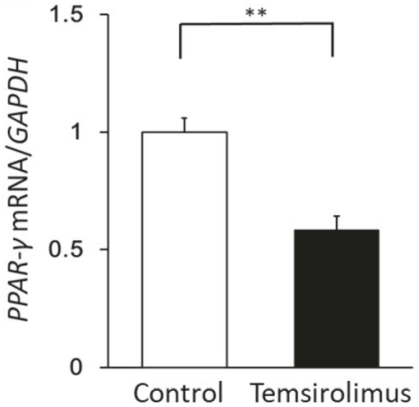

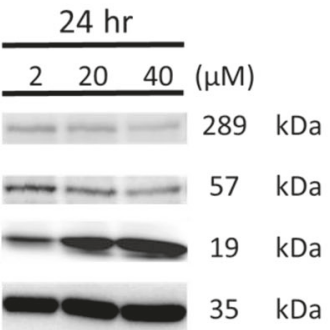

lymphocytic alveolitis with foamy pneumocyte hyperplasia and foamy macrophage accumulation in the alveoli (Table 1, Figs. 2c, d and 3p, q, t).

Since lipids are the main component of alveolar surfactant, homeostasis of the lipid environment in the lung is important for maintaining the lungs. Intrapulmonary lipids are regulated by the following two processes: (a) lipid secretion, reuptake, reutilization, and catabolism by ATII cells and (b) lipid uptake from the systemic circulation [9]. Therefore, dysregulation of lipid metabolism and alteration of fatty acid composition in the alveolar epithelium are known to cause oxidative stress and induce pulmonary inflammation and fibrosis [25, 26]. Furthermore, alteration in alveolar surfactant clearance causes excessive accumulation of surfactant lipids, resulting in pulmonary alveolar proteinosis and alveolar epithelial injury [27]. In an animal study, it was reported that a diet high in saturated fatty acids altered the intrapulmonary lipid composition and induced increased susceptibility to oxygen toxicity [28]. Also, excessive circulating FFAs was suggested to affect the composition and function of alveolar surfactant [14]; intravenous administration of FFAs increased the surfactant pool with pulmonary edema [29]. In human studies, there are some reports that fatty acid intake increases the risk of developing idiopathic pulmonary fibrosis [12], and chronic pulmonary fibrosis is associated with high circulating FFA levels [13]. Therefore, it is suggested that changes in the fatty acid composition of the lung may be associated with lung damage.

Fatty acids within lipid droplets are stored for energy generation and are used as membrane constituents and in steroid hormone biosynthesis. However, lipid droplets are also generated by cellular stress, such as inflammation and oxidative stress [11]. Lipid droplets can be observed in the cytoplasm of pneumocytes [9, 11, 30-32], especially in ATII cells, and it is reported that some drugs, e.g., amiodarone, increased the number of phospholipid-like vacuoles with myelinoid inclusions and enlarged lamellar bodies [33-35]. Moreover, Niemann-Pick disease, which is a lipid storage disorder, and Hermansky-Pudlak syndrome, which is characterized by impaired secretion of surfactant, show both lipid accumulation in the cytoplasm of ATII cells and chronic inflammation and fibrosis of the lung [36-38]. In the present study, we found that the number of lipid-associated spots and the size of round electron-lucent bodies, likely lipid-associated vacuole lipid droplets or degenerated enlarged lamellar bodies within pneumocytes, were significantly more numerous and larger, respectively, in mTORi mice than in BLM mice. However, the level of lung injury in mTORi mice was somewhat mild compared to BLM mice, which are characterized by marked inflammation and fibrosis in the lung [22] (Fig. 4p, q). Because the serum FFA value in mTORi mice was significantly higher than in BLM mice (Fig. 2c), systemic lipid metabolic disorder may affect the characteristic findings of the emergence of multiple lipidassociated vacuoles within pneumocytes following treatment with mTORi. Moreover, we also demonstrated that mTORi administration to MLE-12 cells induces lipidassociated spots in the cytoplasm (Fig. 6a); thus we suggest that the findings of foamy pneumocytes may be associated with systemic lipid abnormality as well as a local effect of lipid abnormality in pneumocytes caused by mTORi. 
Fig. 7 PPAR- $\gamma$ agonist suppresses the effects induced by mTORi administration on a lung epithelial cell line. a Fluorescence staining of MLE12 cells with Nile Red (red) and DAPI (blue) $24 \mathrm{~h}$ after temsirolimus treatment $(20 \mu \mathrm{M})$ with/without pioglitazone $(10 \mu \mathrm{M})$. Scale bars are $5 \mu \mathrm{m} . \mathbf{b}$ Western blot analysis of the expression of cleaved caspase-3 in MLE-12 cells cultured for $24 \mathrm{~h}$ after temsirolimus treatment $(20 \mu \mathrm{M})$ with/without pioglitazone $(10 \mu \mathrm{M})$. c Densitometric analysis of western blot bands, normalized to GAPDH $(n=4)$. Data are 0.05 versus temsirolimus group shown as the mean \pm S.E. $* P<$

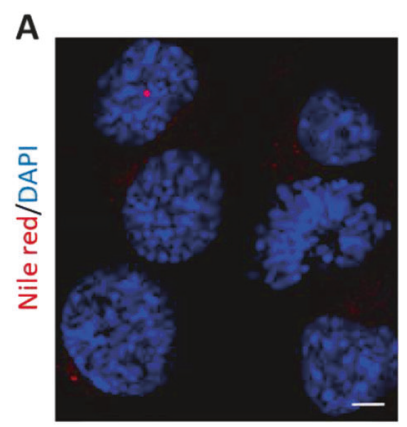

Control

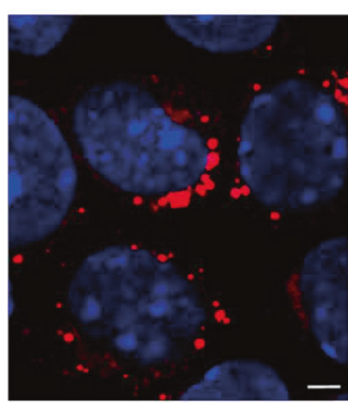

Temsirolimus

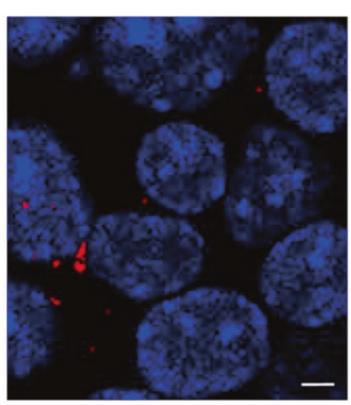

Temsirolimus + Pioglitazone
B

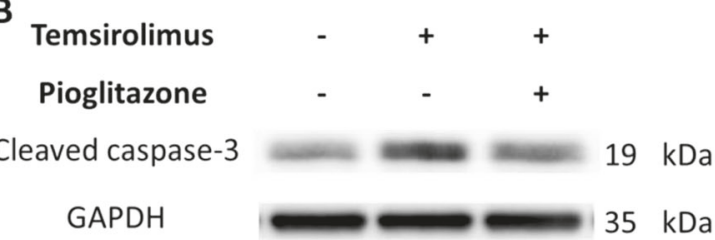

C

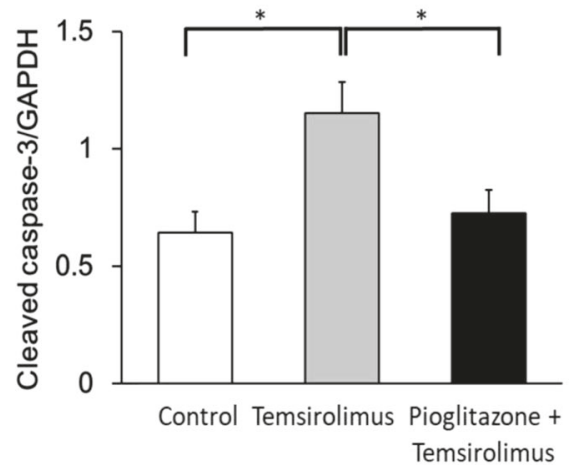

The protein kinase mTOR regulates cellular functions related to the promotion of cell growth and metabolism and forms mTORC1 in its signaling pathway [39]. mTORC1 specifically regulates the expression of PPAR- $\gamma$, which is a nuclear receptor that controls fatty acid synthesis, uptake, and esterification [23]. In the present study, PPAR- $\gamma$ expression in both the mouse lung and lung epithelial cell line was suppressed following mTORi administration (Figs. 5 and 6). These results were consistent with the reported cascade of mTOR [40] and the observation that the expression of PPAR- $\gamma$ was inhibited by administration of the mTOR inhibitor rapamycin. Additionally, we observed elevations in the levels of various inflammatory cytokines such as IL-1 $\beta$, IL-6, MMP-9, CCL2, and TNF- $\alpha$ in mTORi mouse lung (Fig. 5a-e). PPAR- $\gamma$ is known to have protective effects on cell injury through anti-inflammatory and immunomodulatory effects [41], decreasing matrix metalloproteinase activity and inhibiting several cytokines in the alveolar epithelium [42-45]. Thus our findings of lymphocytic alveolitis with increases in various inflammatory cytokines in mTORi mice is likely attributable to the suppressed expression of PPAR- $\gamma$ following mTORi treatment, which is consistent with previous studies of mTORiinduced inflammation that reported leukocyte infiltration in the lung via the production of various cytokines [18, 46-48].

It has been reported that PPAR- $\gamma$ is involved in lipid metabolism of ATII cells and surfactant homeostasis [49, 50] and that PPAR- $\gamma$ repairs deficiencies in fatty-acidbinding proteins, which regulate the synthesis of the main component of surfactant phospholipids [51]. Moreover, deficiency in PPAR- $\gamma$ or its signaling pathway causes the emergence of neutrophils and foamy cells with intraalveolar accumulation of a surfactant-like substance and leads to the induction of inflammatory cytokines [52-54]. Thus it is suggested that mTORi may induce lung injury by the reduction of PPAR- $\gamma$ expression, through alterations in the intrapulmonary surfactant phospholipid environment as well as its general anti-inflammatory effect.

We also showed that treatment with the PPAR- $\gamma$ agonist pioglitazone suppressed the upregulation of lipid-associated spot emergence in the cytoplasm of MLE-12 cells with cleaved caspase- 3 expression following mTORi administration (Fig. 7). It is reported that the PPAR- $\gamma$ agonist 
alleviates lipopolysaccharide-induced apoptosis of ATII cells, through attenuating inflammatory mediators and restoring SP levels [55]. Further, it was reported that a PPAR- $\gamma$ agonist restored surfactant synthesis disorder due to dysfunction of alveolar lipid metabolism in ATII cells [56]. Therefore, the anti-inflammatory and surfactant repair behavior involved in PPAR- $\gamma$ activation by PPAR- $\gamma$ agonist could have influenced the reduction of cleaved caspase-3 expression in MLE-12 cells.

Taken together, it is suggested that mTORi administration likely influences lung injury by reducing antiinflammatory effects and changing the surfactant environment of intrapulmonary lipids through the suppression of PPAR- $\gamma$ expression.

There are several limitations in our study. In particular, the relationship between (1) downregulated PPAR- $\gamma$ expression and (2) pulmonary inflammation, emergence of lipid droplets, and detailed analysis of surfactant in vivo has not been proved directly using our mTORi mouse model with modification of PPAR- $\gamma$ expression. Nevertheless, focusing on the relationship between lipid metabolic stress and alveolar epithelial injury in this mTORi lung injury study represents a potentially novel approach to the study of pulmonary damage in general.

In conclusion, the pathogenesis of mTORi-induced lung disease may involve alveolar epithelial injury via local and systemic lipid metabolic stress with a reduction in PPAR- $\gamma$ expression, which is involved in the homeostasis of the surfactant lipid environment and has anti-inflammatory effects.

Acknowledgements The authors thank Ms. A. Ishikawa, Ms. N. Kuwahara, Ms. K. Wakamasu, Ms. M. Kataoka, and Mr. T. Arai for their expert technical assistance.

Funding This work was supported by JSPS KAKENHI Grant Number JP15K19433 (NK) and by grants-in-aid for scientific research from the Diffuse Lung Diseases Research Group (YT).

\section{Compliance with ethical standards}

Conflict of interest The authors declare that they have no conflict of interest.

Publisher's note: Springer Nature remains neutral with regard to jurisdictional claims in published maps and institutional affiliations.

\section{References}

1. Maroto JP, Hudes G, Dutcher JP, et al. Drug-related pneumonitis in patients with advanced renal cell carcinoma treated with temsirolimus. J Clin Oncol. 2011;29:1750-6.

2. Alexandru S, Ortiz A, Baldovi S, et al. Severe everolimusassociated pneumonitis in a renal transplant recipient. Nephrol Dial Transplant. 2008;23:3353-5.

3. Schrader J, Sterneck M, Klose H, et al. Everolimus-induced pneumonitis: report of the first case in a liver transplant recipient and review of treatment options. Transpl Int. 2010;23:110-3.
4. Carreño CA, Gadea M. Case report of a kidney transplant recipient converted to everolimus due to malignancy: resolution of bronchiolitis obliterans organizing pneumonia without everolimus discontinuation. Transplant Proc. 2007;39:594-5.

5. Saito Y, Kunugi S, Suzuki Y, et al. Granuloma-forming interstitial pneumonia occurring one year after the start of everolimus therapy. Intern Med. 2013;52:263-7.

6. Houde VP, Brûlé S, Festuccia WT, et al. Chronic rapamycin treatment causes glucose intolerance and hyperlipidemia by upregulating hepatic gluconeogenesis and impairing lipid deposition in adipose tissue. Diabetes. 2010;59:1338-48.

7. Sivendran S, Agarwal N, Gartrell B, et al. Metabolic complications with the use of mTOR inhibitors for cancer therapy. Cancer Treat Rev. 2014;40:190-6.

8. Hutson TE, Escudier B, Esteban E, et al. Randomized phase III trial of temsirolimus versus sorafenib as second-line therapy after sunitinib in patients with metastatic renal cell carcinoma. J Clin Oncol. 2014;32:760-7.

9. Plantier L, Besnard V, Xu Y, et al. Activation of sterol-response element-binding proteins (SREBP) in alveolar type II cells enhances lipogenesis causing pulmonary lipotoxicity. J Biol Chem. 2012;287:10099-114.

10. Enzi G, Bevilacqua M, Crepaldi G. Disturbances in pulmonary gaseous exchange in primary hyperlipoproteinemias. Bull Eur Physiopathol Respir. 1976;12:433-42.

11. Lee SJ, Zhang J, Choi AM, et al. Mitochondrial dysfunction induces formation of lipid droplets as a generalized response to stress. Oxid Med Cell Longev. 2013;2013:327167.

12. Miyake Y, Sasaki S, Yokoyama T, et al. Dietary fat and meat intake and idiopathic pulmonary fibrosis: a case-control study in Japan. Int J Tuberc Lung Dis. 2006;10:333-9.

13. Iannello $\mathrm{S}$, Cavaleri A, Camuto $\mathrm{M}$, et al. Low fasting serum triglyceride and high free fatty acid levels in pulmonary fibrosis: a previously unreported finding. MedGenMed. 2002;4:5.

14. Baritussio A, Enzi G, Inelmen EM, et al. Altered surfactant synthesis and function in rats with diet-induced hyperlipidemia. Metabolism. 1980;29:503-10.

15. Müller NL, White DA, Jiang H, et al. Diagnosis and management of drug-associated interstitial lung disease. Br J Cancer. 2004;91 (Suppl 2):S24-S30.

16. Leconte M, Nicco C, Ngô C, et al. The mTOR/AKT inhibitor temsirolimus prevents deep infiltrating endometriosis in mice. Am J Pathol. 2011;179:880-9.

17. Wu L, Birle DC, Tannock IF. Effects of the mammalian target of rapamycin inhibitor CCI-779 used alone or with chemotherapy on human prostate cancer cells and xenografts. Cancer Res. 2005;65:2825-31.

18. Washino S, Ando H, Ushijima K, et al. Temsirolimus induces surfactant lipid accumulation and lung inflammation in mice. Am J Physiol Lung Cell Mol Physiol. 2014;306:L1117-L1128.

19. Harrison JH, Lazo JS. High dose continuous infusion of bleomycin in mice: a new model for drug-induced pulmonary fibrosis. J Pharmacol Exp Ther. 1987;243:1185-94.

20. Murata M, Otsuka M, Mizuno H, et al. Development of an enzyme-linked immunosorbent assay for measurement of rat pulmonary surfactant protein D using monoclonal antibodies. Exp Lung Res. 2010;36:463-8.

21. Livak KJ, Schmittgen TD. Analysis of relative gene expression data using real-time quantitative PCR and the 2(-Delta Delta C(T)) Method. Methods. 2001;25:402-8.

22. Thrall RS, McCormick JR, Jack RM, et al. Bleomycin-induced pulmonary fibrosis in the rat: inhibition by indomethacin. Am J Pathol. 1979;95:117-30.

23. Laplante M, Sabatini DM. Regulation of mTORC1 and its impact on gene expression at a glance. J Cell Sci. 2013; 126:1713-9. 
24. Wikenheiser KA, Vorbroker DK, Rice WR, et al. Production of immortalized distal respiratory epithelial cell lines from surfactant protein $\mathrm{C} /$ simian virus 40 large tumor antigen transgenic mice. Proc Natl Acad Sci USA. 1993;90:11029-33.

25. Whitsett JA, Wert SE, Weaver TE. Alveolar surfactant homeostasis and the pathogenesis of pulmonary disease. Annu Rev Med. 2010;61:105-19.

26. Sunaga $\mathrm{H}$, Matsui $\mathrm{H}$, Ueno $\mathrm{M}$, et al. Deranged fatty acid composition causes pulmonary fibrosis in Elovl6-deficient mice. Nat Commun. 2013;4:2563.

27. Borie R, Danel C, Debray MP, et al. Pulmonary alveolar proteinosis. Eur Respir Rev. 2011;20:98-107.

28. Kehrer JP, Autor AP. The effect of dietary fatty acids on the composition of adult rat lung lipids: relationship to oxygen toxicity. Toxicol Appl Pharmacol. 1978;44:423-30.

29. Oyarzun MJ, Cabezas E, Donoso P, et al. Effects of free fatty acid infusion on rabbit pulmonary surfactant. Influence of corticosteroids. Bull Eur Physiopathol Respir. 1984;20:105-11.

30. Fang Y, Wang S, Zhu T, et al. Atherogenic high cholesterol/high fat diet induces TLRs-associated pulmonary inflammation in C57BL/6J mice. Inflamm Res. 2017;66:39-47.

31. McNeish J, Aiello RJ, Guyot D, et al. High density lipoprotein deficiency and foam cell accumulation in mice with targeted disruption of ATP-binding cassette transporter-1. Proc Natl Acad Sci USA. 2000;97:4245-50.

32. Richmond VL, Chi EY. Ultrastructural observations in copperdeficient guinea-pig lung cells. Int J Exp Pathol. 1993;74:133-43.

33. Bedrossian CW, Warren CJ, Ohar J, et al. Amiodarone pulmonary toxicity: cytopathology, ultrastructure, and immunocytochemistry. Ann Diagn Pathol. 1997;1:47-56.

34. Zhang M, Xie Y, Yan R, et al. Curcumin ameliorates alveolar epithelial injury in a rat model of chronic obstructive pulmonary disease. Life Sci. 2016;164:1-8.

35. Kumar RK, Truscott JY, Rhodes GC, et al. Type 2 pneumocyte responses to cyclophosphamide-induced pulmonary injury: functional and morphological correlation. Br J Exp Pathol. 1988; 69:69-80.

36. Chung MJ, Lee KS, Franquet T, et al. Metabolic lung disease: imaging and histopathologic findings. Eur J Radiol. 2005; $54: 233-45$.

37. Nicholson AG, Florio R, Hansell DM, et al. Pulmonary involvement by Niemann-Pick disease. A report of six cases. Histopathology. 2006;48:596-603.

38. Nakatani Y, Nakamura N, Sano J, et al. Interstitial pneumonia in Hermansky-Pudlak syndrome: significance of florid foamy swelling/degeneration (giant lamellar body degeneration) of type-2 pneumocytes. Virchows Arch. 2000;437:304-13.

39. Yang H, Rudge DG, Koos JD, et al. mTOR kinase structure, mechanism and regulation. Nature. 2013;497:217-23.

40. Kim JE, Chen J. regulation of peroxisome proliferator-activated receptor-gamma activity by mammalian target of rapamycin and amino acids in adipogenesis. Diabetes. 2004;53:2748-56.

41. Belvisi MG, Hele DJ. Peroxisome proliferator-activated receptors as novel targets in lung disease. Chest. 2008;134:152-7.
42. Varisco BM, Ambalavanan N, Whitsett JA, et al. Thy-1 signals through PPAR $\gamma$ to promote lipofibroblast differentiation in the developing lung. Am J Respir Cell Mol Biol. 2012;46:765-72.

43. Burgess HA, Daugherty LE, Thatcher TH, et al. PPARgamma agonists inhibit TGF-beta induced pulmonary myofibroblast differentiation and collagen production: implications for therapy of lung fibrosis. Am J Physiol Lung Cell Mol Physiol. 2005;288: L1146-L1153.

44. Ferguson HE, Kulkarni A, Lehmann GM, et al. Electrophilic peroxisome proliferator-activated receptor-gamma ligands have potent antifibrotic effects in human lung fibroblasts. Am J Respir Cell Mol Biol. 2009;41:722-30.

45. Belvisi MG, Hele DJ, Birrell MA. Peroxisome proliferatoractivated receptor gamma agonists as therapy for chronic airway inflammation. Eur J Pharmacol. 2006;533:101-9.

46. Minhajuddin M, Fazal F, Bijli KM, et al. Inhibition of mammalian target of rapamycin potentiates thrombin-induced intercellular adhesion molecule-1 expression by accelerating and stabilizing NF-kappa B activation in endothelial cells. J Immunol. 2005;174:5823-9.

47. Kezic A, Becker JU, Thaiss F. The effect of mTOR-inhibition on $\mathrm{NF}-\kappa \mathrm{B}$ activity in kidney ischemia-reperfusion injury in mice. Transplant Proc. 2013;45:1708-14.

48. Willemsen AE, Grutters JC, Gerritsen WR, et al. mTOR inhibitorinduced interstitial lung disease in cancer patients: comprehensive review and a practical management algorithm. Int $\mathrm{J}$ Cancer. 2016;138:2312-21.

49. Chen H, Jackson S, Doro M, et al. Perinatal expression of genes that may participate in lipid metabolism by lipid-laden lung fibroblasts. J Lipid Res. 1998;39:2483-92.

50. Malur A, Baker AD, McCoy AJ, et al. Restoration of PPAR $\gamma$ reverses lipid accumulation in alveolar macrophages of GM-CSF knockout mice. Am J Physiol Lung Cell Mol Physiol. 2011;300: L73-L80.

51. Guthmann F, Schachtrup C, Tölle A, et al. Phenotype of palmitic acid transport and of signalling in alveolar type II cells from E/HFABP double-knockout mice: contribution of caveolin-1 and PPARgamma. Biochim Biophys Acta. 2004;1636:196-204.

52. Baker AD, Malur A, Barna BP, et al. Targeted PPAR \{gamma\} deficiency in alveolar macrophages disrupts surfactant catabolism. J Lipid Res. 2010;51:1325-31.

53. Reddy RC, Keshamouni VG, Jaigirdar SH, et al. Deactivation of murine alveolar macrophages by peroxisome proliferatoractivated receptor-gamma ligands. Am J Physiol Lung Cell Mol Physiol. 2004;286:L613-L619.

54. Malur A, Mccoy AJ, Arce S, et al. Deletion of PPAR gamma in alveolar macrophages is associated with a Th-1 pulmonary inflammatory response. J Immunol. 2009;182:5816-22.

55. Xiao B, Xu J, Wang G, et al. Troglitazone-activated PPAR $\gamma$ inhibits LPS-induced lung alveolar type II epithelial cells injuries via TNF- $\alpha$. Mol Biol Rep. 2011;38:5009-15.

56. Schachtrup C, Malcharek S, Haitsma JJ, et al. Activation of PPARgamma reverses a defect of surfactant synthesis in mice lacking two types of fatty acid binding protein. Biochim Biophys Acta. 2008;1781:314-20. 